\title{
Bernoulli Wavelets Operational Matrices Method for the Solution of Nonlinear Stochastic Itô-Volterra Integral Equations
}

\author{
S. C. Shiralashetti ${ }^{1, *}$ and Lata Lamani ${ }^{2}$ \\ ${ }^{1}$ Department of Mathematics, Karnatak University, Dharwad, India \\ e-mail: scshiralashetti@kud.ac.in \\ ${ }^{2}$ Department of Mathematics, Karnatak University, Dharwad, India \\ e-mail: latalamani@gmail.com
}

\begin{abstract}
This article gives an effective strategy to solve nonlinear stochastic Itô-Volterra integral equations (NSIVIE). These equations can be reduced to a system of nonlinear algebraic equations with unknown coefficients, using Bernoulli wavelets, their operational matrix of integration (OMI), stochastic operational matrix of integration (SOMI) and these equations can be solved numerically. Error analysis of the proposed method is given. Moreover, the results obtained are compared to exact solutions with numerical examples to show that the method described is accurate and precise.
\end{abstract}

\section{Introduction}

Wavelets are mathematical functions that isolate the data and analyze each variable with the corresponding resolution in various frequency components. As a mathematical tool, wavelet can be used to extract information from the different forms of data, including, seismic waves, earthquakes, music, image processing, signal processing, acoustics, nuclear engineering, and astronomy.

We lack enough knowledge in certain issues related to behavioral analysis for some processes to decide how it conforms, or its operation is so complex that it is irrelevant or difficult to explain precisely. In such a scenario a probabilistic model is always helpful. Due to their significance in modeling, science and technological phenomenon, nonlinear stochastic and deterministic functions have been widely investigated and studied. Received: October 3, 2020; Accepted: November 2, 2020

2010 Mathematics Subject Classification: 60H05, 60H35, 65T60.

Keywords and phrases: nonlinear stochastic Itô-Volterra integral equations, Bernoulli wavelets, Bernoulli polynomials, Brownian motion. 
Stochastic integral equations are used in simulation of many physical, science and engineering phenomena. In recent years, as can be seen in some situations, the complex hierarchical systems of physics, medicines, engineering and finance have been extremely challenging [1-9]. Within these systems a source of noise is generally governed by likelihood rules. The simulations of these phenomena involve various stochastic integral equations, differential equations and so on [10-16]. These problems are also hard to solve. Therefore, numerical approaches become important to obtain approximate solutions [17-19].

The Itô integral typically used in applied mathematics is named after Kiyoshi Itô. In analytical forms, stochastic integral equations cannot be resolved normally and therefore a necessary prerequisite for their solution in applied mathematics become necessary. Throughout recent years, the computational methods such as [20-24] have been used to solve stochastic integral equations. However, very few articles exist on stochastic integral equations. The operational matrix method using Bernoulli wavelets for solving linear ItôVolterra integral equations was recently employed by Mirzaee and Samadyar [25]. This article however attempts to frame a stochastic operational matrix of integration of Bernoulli wavelets (SOMIBW) and is employed to obtain the solution to the particular case of the NSIVIE as follows

$$
y(x)=f(x)+\int_{0}^{x} k_{1}(x, t) \mu(y(t)) d t+\int_{0}^{x} k_{2}(x, t) \sigma(y(t)) d W(t),
$$

where $x \in[0,1), k_{1}(x, t)$ and $k_{2}(x, t)$ are functions of $x$ and $t, \mu(y(x))$ and $\sigma(y(x))$ are known functions, $y(x)$ is the unknown that is to be determined and $W(x)$ is the Brownian motion process defined on probability space $(\Omega, F, P)$ that consists of the sample space $\Omega$, a $\sigma$-algebra $F$ of subsets of $\Omega$ which we call events, and a real-valued set function $P$ defined on $F$ that is called probability.

Equation (1) appears in various fields including engineering, mathematics, biology, health and social science. It is very hard or even difficult to solve this equation, so we develop an efficient method for solving it. In this article, equation (1) is numerically solved by the use of operational matrix of integration (OMI) and stochastic OMI based on Bernoulli wavelets. This equation is reduced to a nonlinear system of algebraic equations by the use of collocation points and these matrices can be solved by an effective numerical method such as Newton's method. 
The rest of the work is structured accordingly. Section 2 provides some basic definitions and characteristics of stochastic calculus, wavelets, and Bernoulli wavelets. Also, in this section, OMI and stochastic OMI based on Bernoulli wavelets are obtained. The proposed method of solution is given to estimate the solution of non linear ItôVolterra integral equation in Section 3. In Section 4, Numerical examples are presented to show the efficiency and reliability of the proposed method. Finally, the conclusion of the article is given in Section 5.

\section{Properties of Stochastic Calculus, Wavelets, and Bernoulli Wavelets}

\subsection{Brownian motion}

Definition 2.1. A stochastic process, $\{W(x): 0 \leq x<\infty\}$, is called a standard Brownian motion if

- $W(0)=0$

- The stochastic process $\{W(x): 0 \leq x<\infty\}$ has continuous sample paths.

- The process has independent, stationary increments.

Definition 2.2. An $n$-dimensional process, $W(x)=\left(W(x)^{1}, \ldots, W(x)^{n}\right)$, is a standard $n$-dimensional Brownian motion if each $W(x)^{i}$ is a standard Brownian motion and the $W(x)^{i}$, s are independent of each other.

\subsection{Wavelets}

A family of functions is generated by mother wavelets by dilating and translating itself, which we call wavelets. The wavelet family is as given below [26]:

$$
\psi_{a, b}(x)=|a| \frac{-1}{2} \psi\left(\frac{x-b}{a}\right), \quad a, b \in R, a \neq 0,
$$

where $a$ and $b$ are denoted respectively for the dilation parameter and translation parameter. Nevertheless, $b$ varies continously.

If we let $a=a_{0}^{-k}, b=n a_{0}^{-k} b_{0}, a_{0}>1$ and $b_{0}>1$, where, $n, k \in N$, the family of discrete wavelets may be given as,

$$
\psi_{k, n}(x)=\left|a_{0}\right| \frac{k}{2} \psi\left(a_{0}^{k} x-n b_{0}\right), \quad a, b \in R, a \neq 0,
$$

where the wavelet bases in $L^{2}(R)$ are $\psi_{k, n}(x)$. 


\subsection{Bernoulli polynomials}

Bernoulli polynomials are defined in general as [25]:

$$
B_{m}(x)=\sum_{i=0}^{m}\left(\begin{array}{c}
m \\
i
\end{array}\right) \alpha_{m-i} x^{i}, \quad m=0,1,2, \ldots,
$$

where $\alpha_{i}, i=0, \ldots, m$ are Bernoulli numbers. For instance, starting five Bernoulli numbers are:

$$
\alpha_{0}=1, \quad \alpha_{1}=-\frac{1}{2}, \quad \alpha_{2}(x)=\frac{1}{6}, \quad \alpha_{3}(x)=0, \quad \alpha_{4}(x)=-\frac{1}{30} .
$$

And the first four Bernoulli polynomials are:

$$
B_{0}(x)=1, \quad B_{1}(x)=x-\frac{1}{2}, \quad B_{2}(x)=x^{2}-x+\frac{1}{6}, \quad B_{3}(x)=x^{3}-\frac{3}{2} x^{2}+\frac{1}{2} x .
$$

\subsection{Bernoulli wavelets}

Bernoulli wavelets are defined as follows [25]:

$$
\psi_{n, m}(x)= \begin{cases}2^{\frac{k-1}{2}} \bar{B}_{m}\left(2^{k-1} x-n+1\right), & \frac{n-1}{2^{k-1}} \leq x<\frac{n}{2^{k-1}}, \\ 0, & \text { otherwise, }\end{cases}
$$

in which

$$
\bar{B}_{m}(x)= \begin{cases}1, & m=0 \\ \frac{1}{\sqrt{\left(\left((-1)^{m-1}(m !)^{2}\right) /((2 m) !)\right) \alpha_{2 m}}} B_{m}(x), & m>0\end{cases}
$$

where, $m=0,1, \ldots, M-1$ denotes the order of the Bernoulli polynomials and $n=1,2, \ldots, 2^{k-1}, k \in N$.

For instance, for $k=2$ and $M=2$, we get

$$
\begin{aligned}
& \left.\begin{array}{l}
\psi_{10}(x)=\sqrt{2} \\
\psi_{11}(x)=\sqrt{6}(4 x-1)
\end{array}\right\} \quad 0 \leq x<\frac{1}{2}, \\
& \left.\begin{array}{l}
\psi_{10}(x)=\sqrt{2} \\
\psi_{11}(x)=\sqrt{6}(4 x-3)
\end{array}\right\} \quad \frac{1}{2} \leq x<1 .
\end{aligned}
$$




\subsection{Approximation of function}

Suppose $f(x) \in L^{2}[0,1)$ is expanded in terms of the Bernoulli wavelets as

$$
f(x)=\sum_{n=1}^{\infty} \sum_{m=0}^{\infty} c_{n m} \psi_{n m}(x)
$$

Truncating the above infinite series, we get

$$
f(x) \simeq \sum_{n=1}^{2^{k-1}} \sum_{m=0}^{M-1} c_{n m} \psi_{n m}(x)=C^{T} \psi(x)=f_{\hat{m}}(x),
$$

where, $C$ and $\psi(x)$ are $\hat{m} \times 1\left(\hat{m}=2^{k-1} M\right)$ matrices given by

$$
\begin{gathered}
C=\left[c_{10}, c_{11}, \ldots, c_{1, M-1}, c_{20}, \ldots, c_{2, M-1}, \ldots, c_{2^{k-1}, 0}, \ldots, c_{2^{k-1}, M-1}\right]^{T}, \\
\psi(x)=\left[\psi_{10}(x), \psi_{11}(x), \ldots, \psi_{1, M-1}(x), \psi_{20}(x), \ldots, \psi_{2, M-1}(x), \ldots,\right. \\
\left.\Psi_{2^{k-1}, 0}(x), \ldots, \psi_{2^{k-1}, M-1}(x)\right]^{T} .
\end{gathered}
$$

\subsection{OMI and stochastic OMI of Bernoulli wavelets (SOMIBW)}

OMI of Bernoulli wavelets is given in detail in [25]. Now, we derive the stochastic OMI of Bernoulli wavelets as follows:

The stochastic integral of $\psi(x)$ can be obtained as follows:

$$
\int_{0}^{x} \psi(t) d W(t)=P_{s} \psi(x)
$$

where $P_{S}$ is a $\hat{m} \times \hat{m}$ matrix and is called the SOMIBW. In particular, for $M=2$ and $k=2$, we have

$$
\int_{0}^{x} \psi_{10}(t) d W(t)=\left\{\begin{array}{ll}
\sqrt{2} W(x), & 0 \leq x<1 / 2 \\
\sqrt{2} W\left(\frac{1}{2}\right), & 1 / 2 \leq x<1
\end{array} \simeq W\left(\frac{1}{4}\right) \psi_{10}(x)+W\left(\frac{1}{2}\right) \psi_{20}(x),\right.
$$




$$
\begin{aligned}
& \int_{0}^{x} \psi_{11}(t) d W(t)= \begin{cases}\left(4 \sqrt{6} x W(x)-\sqrt{6} W(x)-\int_{0}^{x} 4 \sqrt{6} W(t) d t\right), & 0 \leq x<1 / 2 \\
\sqrt{6}\left(W\left(\frac{1}{2}\right)-4 \int_{0}^{1 / 2} W(t) d t\right), & 1 / 2 \leq x<1\end{cases} \\
& \simeq\left(\frac{-1}{\sqrt{2}} \int_{0}^{1 / 4} 4 \sqrt{6} W(t) d t\right) \psi_{10}(x)+W\left(\frac{1}{4}\right) \psi_{11}(x) \\
& +\left(\frac{\sqrt{6} W\left(\frac{1}{2}\right)-\int_{0}^{1 / 2} 4 \sqrt{6} W(t) d t}{\sqrt{2}}\right) \psi_{20}(x) \\
& \int_{0}^{x} \psi_{20}(t) d W(t)=\left\{\begin{array}{ll}
0, & 0 \leq x<1 / 2 \\
\sqrt{2}\left(W(x)-W\left(\frac{1}{2}\right)\right), & 1 / 2 \leq x<1
\end{array} \simeq\left(W\left(\frac{3}{4}\right)-W\left(\frac{1}{2}\right)\right) \psi_{20}(x),\right. \\
& \int_{0}^{x} \psi_{21}(t) d W(t)= \begin{cases}0, & 0 \leq x<1 / 2 \\
(4 \sqrt{6} x-3 \sqrt{6}) W(x)+\sqrt{6} W\left(\frac{1}{2}\right)-\int_{1 / 2}^{x} 4 \sqrt{6} W(t) d t, & 1 / 2 \leq x<1\end{cases} \\
& \simeq\left(\frac{\sqrt{6} W\left(\frac{1}{2}\right)-\int_{1 / 2}^{3 / 4} 4 \sqrt{6} W(t) d t}{\sqrt{2}}\right) \psi_{20}(x)+W\left(\frac{3}{4}\right) \psi_{21}(x),
\end{aligned}
$$

Using equations (9) to (12), we get

$$
\int_{0}^{x} \psi(t) d W(t)=\left[\begin{array}{l}
\int_{0}^{x} \psi_{10} d W(t) \\
\int_{0}^{x} \psi_{11} d W(t) \\
\int_{0}^{x} \psi_{12} d W(t) \\
\int_{0}^{x} \psi_{20} d W(t)
\end{array}\right] .
$$


Therefore,

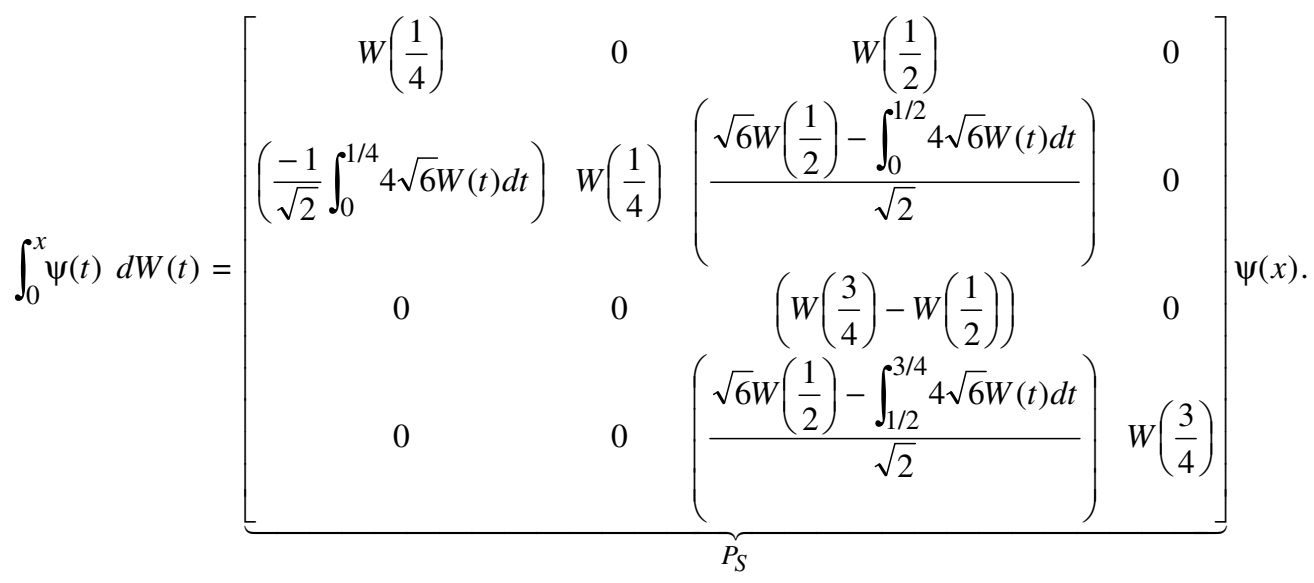

The SOMIBW is derived here for $k=2$ and $M=2$ i.e. for $\hat{m}=4$ and the same can be extended for different values of $k$ and $M$ i.e. for different values of $\hat{m}$.

Remark 1. If $F$ is a $\hat{m}$-vector, then

$$
\psi(x) \psi^{T}(x) F=\tilde{F} \psi(x),
$$

where, $\psi(x)$ is the Bernoulli wavelet coefficient matrix and $\tilde{F}$ is a $\hat{m} \times \hat{m}$ matrix given by

$$
\tilde{F}=\psi(x) \bar{F} \psi^{-1}(x)
$$

where $\bar{F}=\operatorname{diag}\left(\psi^{-1}(x) F\right)$. Also, for a $\hat{m} \times \hat{m}$ matrix $C$,

$$
\psi^{T}(x) C \psi(x)=\hat{C}^{T} \psi(x)
$$

in which, $X=\operatorname{diag}\left(\psi^{T}(x) C \psi(x)\right)$ is a $\hat{m}$-vector and $\hat{C}^{T}=X \psi^{-1}(x)$.

Remark 2. If $\mu$ is an analytic function on $R$ and $C^{T} \psi(x)$ be the the expansion of $f(x)$ in terms of Bernoulli wavelets, where $C$ is given in equation (6), then

$$
\mu(f(x)) \simeq \mu\left(C^{T}\right) \psi(x),
$$

where $\mu\left(C^{T}\right)=\left[\begin{array}{llll}\mu\left(c_{1}\right) & \mu\left(c_{2}\right) & \cdots & \mu\left(c_{\hat{m}}\right)\end{array}\right]$. 
Remark 3. If $\mu$ is an analytic function on $R$ and $C^{T} \psi(x)$ be the the expansion of $f(x)$ in terms of Bernoulli wavelets, where $C$ is given in equation (6), then

$$
\mu(f(x)) \simeq \mu\left(\tilde{C}^{T}\right) \psi^{-1}(x) \psi(x),
$$

where $\tilde{C}^{T}=C^{T} \psi(x), \psi(x)$ is the Bernoulli wavelets coefficient matrix given in (7) and $\mu\left(C^{T}\right)$ is given in Remark 2 .

\section{Bernoulli Wavelets Method of Solution (BWM)}

In this section, we use the newly derived SOMIBW for the numerical solution of NSIVIE. Here we consider the equation in (1) as:

$$
y(x)=f(x)+\int_{0}^{x} k_{1}(x, t) \mu(y(t)) d t+\int_{0}^{x} k_{2}(x, t) \sigma(y(t)) d W(t) .
$$

Approximating $f(x), y(x)$ and $k_{i}(x, t), i=1,2$ with respect to Bernoulli wavelets as follows:

$$
y(x) \simeq C^{T} \psi(x)=C \psi^{T}(x),
$$

where $C$ is given in equation (6) and is the unknown vector to be determined.

$$
\begin{gathered}
f(x) \simeq F^{T} \psi(x)=F \psi^{T}(x), \\
k_{1}(x, t) \simeq \psi^{T}(x) K_{1} \psi(t)=\psi^{T}(t) K_{1}^{T} \psi(x), \\
k_{2}(x, t) \simeq \psi^{T}(x) K_{2} \psi(t)=\psi^{T}(t) K_{2}^{T} \psi(x),
\end{gathered}
$$

where $C$ and $F$ are Bernoulli wavelet coefficient vectors and $K_{1}$ and $K_{2}$ are Bernoulli wavelet matrices. Substituting (19), (20), (21) and (22) in (18), we get

$$
\begin{aligned}
C^{T} \psi(x) \simeq & F^{T} \psi(x)+\psi^{T}(x) K_{1}\left(\int_{0}^{x} \psi(t) \mu\left(C^{T} \psi(t)\right) d t\right) \\
& +\psi^{T}(x) K_{2}\left(\int_{0}^{x} \psi(t) \sigma\left(C^{T} \psi(t)\right) d W(t)\right) .
\end{aligned}
$$

Now, by using Remark 3, equation (23) can be rewritten as, 


$$
\begin{aligned}
C^{T} \psi(x) \simeq & F^{T} \psi(x)+\psi^{T}(x) K_{1}\left(\int_{0}^{x} \psi(t) \psi^{T}(t) X_{1} d t\right) \\
& +\psi^{T}(x) K_{2}\left(\int_{0}^{x} \psi(t) \psi^{T}(t) X_{2} d W(t)\right),
\end{aligned}
$$

where $\quad X_{1}^{T}=\mu\left(\tilde{C}^{T}\right) \psi^{-1}(x), \quad X_{2}{ }^{T}=\sigma\left(\tilde{C}^{T}\right) \psi^{-1}(x) \quad$ and $\quad \tilde{C}^{T}=C^{T} \psi(x)$. Using equation (24) and Remark 1, we get

$$
\begin{aligned}
C^{T} \psi(x) \simeq & F^{T} \psi(x)+\psi^{T}(x) K_{1}\left(\int_{0}^{x} \tilde{X}_{1} \psi(t) d t\right) \\
& +\psi^{T}(x) K_{2}\left(\int_{0}^{x} \tilde{X}_{2} \psi(t) d W(t)\right),
\end{aligned}
$$

where $\tilde{X}_{1}$ and $\tilde{X}_{2}$ are $\hat{m} \times \hat{m}$ matrices described in Remark 1. Applying the OMI of Bernoulli wavelets $P$ explained in [25] and the stochastic OMI of Bernoulli wavelets described in Section 2, equation (25) reduces to:

$$
C^{T} \psi(x) \simeq F^{T} \psi(x)+\psi^{T}(x) K_{1} \tilde{X}_{1} P \psi(x)+\psi^{T}(x) K_{2} \tilde{X}_{2} P_{S} \psi(x) .
$$

Let us assume that $\delta_{1}=K_{1} \tilde{X}_{1} P$ and $\delta_{2}=K_{2} \tilde{X}_{2} P_{S}$. Again using Remark 1, equation (26) reduces to

$$
C^{T} \psi(x)-\hat{\delta}_{1} \psi(x)-\hat{\delta}_{2} \psi(x) \simeq F^{T} \psi(x),
$$

where $\hat{\delta}_{1}$ and $\hat{\delta}_{2}$ are $\hat{m}$-vectors containing a nonlinear combination of elements of $C$. Equation (27) holds for all $x \in[0,1)$. Replacing $\simeq$ by $=$, equation (27) reduces to a nonlinear system of equations $C^{T}-\hat{\delta}_{1}-\hat{\delta}_{2} \simeq F^{T}$. Solving this nonlinear system, we get the unknown vector $C$. Substituting this obtained vector in equation (19), we obtain the solution of NSIVIE (1).

\section{Numerical Experiments}

Test problem 1. Consider the SLSVIE [27],

$$
y(x)=\frac{1}{20}+\int_{0}^{x}\left(\frac{1}{100} y(t)+\frac{1}{32} y^{2}(t)\right) d t+\frac{1}{8} \int_{0}^{x} y(t) d W(t) .
$$


where the exact solution of this problem is found to be

$$
y(x)=\frac{\exp \left(\left(\frac{1}{100}-\frac{(1 / 8)^{2}}{2}\right) x+\frac{1}{8} W(x)\right)}{\frac{1}{20}-\frac{1}{32} \int_{0}^{x} \exp \left(\left(\frac{1}{100}-\frac{(1 / 8)^{2}}{2}\right) t+\frac{1}{8} W(t)\right) d t},
$$

where $y(x)$ is the unknown stochastic process defined on the probability space $(\Omega, F, P)$, and $W(x)$ is the Brownian motion process. Table 1 shows the numerical results obtained by the method described in Section 3 (BWM), exact solution and absolute errors (AE) for $k=2, \quad M=3$ and $k=3, \quad M=2$, Table 2 shows the comparison of absolute errors of test problem 1 for different values of $k$ and $M$ and Figure 1 shows the graph of exact and approximate values of test problem 1 for $k=3$ and $M=2$.

Table 1. Comparison of exact, Bernoulli wavelet solution (BWS), and AE for test problem 1 .

\begin{tabular}{|c|c|c|c|c|c|c|}
\hline \multirow{2}{*}{$t$} & \multicolumn{3}{|c|}{$k=2$ and $M=3$} & \multicolumn{3}{c|}{$k=3$ and $M=2$} \\
\cline { 2 - 7 } & $\begin{array}{c}\text { Exact } \\
\text { solution }\end{array}$ & $B W S$ & $A E$ & $\begin{array}{c}\text { Exact } \\
\text { solution }\end{array}$ & $B W S$ & $A E$ \\
\hline 0 & 0.0500 & 0.0556 & $5.5802 \mathrm{e}-03$ & 0.0500 & 0.0566 & $6.6481 \mathrm{e}-03$ \\
\hline 0.1 & 0.0464 & 0.0526 & $6.1972 \mathrm{e}-03$ & 0.0501 & 0.0521 & $1.9900 \mathrm{e}-03$ \\
\hline 0.2 & 0.0509 & 0.0530 & $2.0964 \mathrm{e}-03$ & 0.0511 & 0.0515 & $4.0000 \mathrm{e}-04$ \\
\hline 0.3 & 0.0524 & 0.0518 & $5.6708 \mathrm{e}-04$ & 0.0479 & 0.0451 & $2.8000 \mathrm{e}-03$ \\
\hline 0.4 & 0.0508 & 0.0490 & $1.7932 \mathrm{e}-03$ & 0.0500 & 0.0444 & $5.5800 \mathrm{e}-03$ \\
\hline 0.5 & 0.0540 & 0.0469 & $7.1460 \mathrm{e}-03$ & 0.0469 & 0.0466 & $3.0000 \mathrm{e}-04$ \\
\hline 0.6 & 0.0561 & 0.0458 & $1.0322 \mathrm{e}-02$ & 0.0428 & 0.0489 & $6.1200 \mathrm{e}-03$ \\
\hline 0.7 & 0.0473 & 0.0488 & $1.4846 \mathrm{e}-03$ & 0.0427 & 0.0486 & $5.9400 \mathrm{e}-03$ \\
\hline 0.8 & 0.0454 & 0.0478 & $2.4723 \mathrm{e}-03$ & 0.0427 & 0.0450 & $2.2600 \mathrm{e}-03$ \\
\hline 0.9 & 0.0503 & 0.0429 & $7.3592 \mathrm{e}-03$ & 0.0428 & 0.0446 & $1.8700 \mathrm{e}-03$ \\
\hline
\end{tabular}


Table 2. Comparison of absolute errors of test problem 1 for different values of $k$ and $M$.

\begin{tabular}{cc}
\hline$B W S$ & Maximum absolute error $\left(E_{m}\right)$ \\
\hline$k=2, M=3$ & $1.0322 \mathrm{e}-02$ \\
$k=3, M=2$ & $6.6481 \mathrm{e}-03$ \\
\hline
\end{tabular}

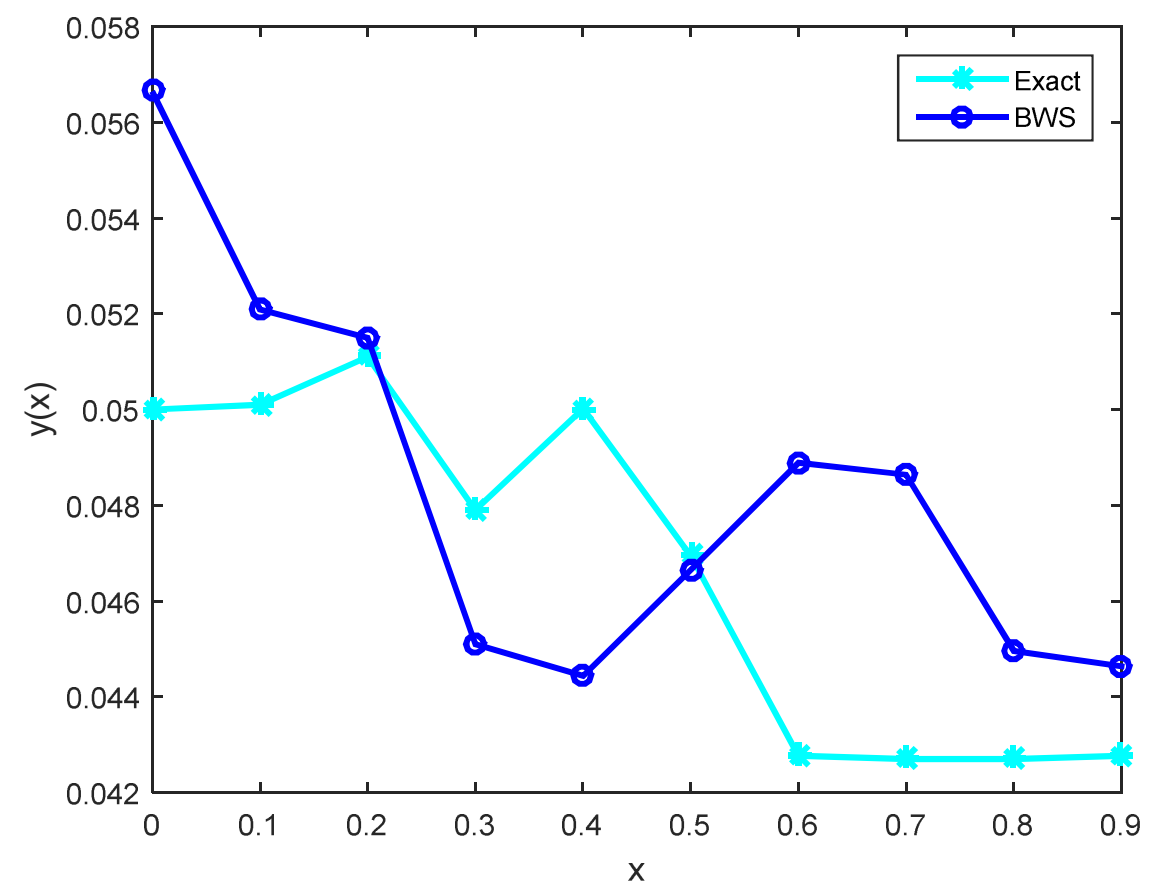

Figure 1. Graph of exact and BWS of test problem 1 for $k=3$ and $M=2$.

Test problem 2. Consider the SLSVIE [27],

$$
y(x)=\frac{1}{20}-\frac{1}{16} \int_{0}^{x} \sin (y(t)) \cos ^{3}(y(t)) d t+\frac{1}{4} \int_{0}^{x} \cos ^{2}(y(t)) d W(t) .
$$

where the exact solution of this problem is found to be

$$
y(x)=\arctan \left(\frac{1}{4} W(x)+\tan \left(\frac{1}{20}\right)\right),
$$

where $y(x)$ is the unknown stochastic process defined on the probability space 
$(\Omega, F, P)$, and $W(x)$ is the Brownian motion process. Table 3 shows the numerical results obtained by the method described in Section 3 (BWM), exact solution and absolute errors (AE) for $k=2, \quad M=3$ and $k=3, \quad M=2$, Table 4 shows the comparison of absolute errors of test problem 2 for different values of $k$ and $M$ and Figure 2 shows the graph of exact and approximate values of test problem 2 for $k=3$ and $M=2$.

Table 3. Comparison of exact, Bernoulli wavelet solution (BWS), and AE for test problem 2 .

\begin{tabular}{|c|c|c|c|c|c|c|}
\hline \multirow{2}{*}{$t$} & \multicolumn{3}{|c|}{$k=2$ and $M=3$} & \multicolumn{3}{c|}{$k=3$ and $M=2$} \\
\cline { 2 - 7 } & $\begin{array}{c}\text { Exact } \\
\text { solution }\end{array}$ & $B W S$ & $A E$ & $\begin{array}{c}\text { Exact } \\
\text { solution }\end{array}$ & $B W S$ & $A E$ \\
\hline 0 & 0.0500 & 0.2351 & $1.8509 \mathrm{e}-01$ & 0.0500 & 0.3160 & $2.6600 \mathrm{e}-01$ \\
\hline 0.1 & 0.1016 & 0.0611 & $4.0454 \mathrm{e}-02$ & 0.0853 & 0.1165 & $3.1150 \mathrm{e}-02$ \\
\hline 0.2 & 0.0691 & -0.0184 & $8.7485 \mathrm{e}-02$ & 0.0652 & 0.0795 & $1.4300 \mathrm{e}-02$ \\
\hline 0.3 & 0.0971 & -0.0986 & $1.9570 \mathrm{e}-01$ & 0.1678 & -0.1963 & $3.6410 \mathrm{e}-01$ \\
\hline 0.4 & 0.1856 & -0.1795 & $3.6511 \mathrm{e}-01$ & 0.1630 & -0.2265 & $3.8949 \mathrm{e}-01$ \\
\hline 0.5 & 0.1860 & -0.1437 & $3.2965 \mathrm{e}-01$ & 0.0458 & -0.1429 & $1.8865 \mathrm{e}-01$ \\
\hline 0.6 & 0.1463 & -0.0943 & $2.4053 \mathrm{e}-01$ & 0.0659 & -0.0605 & $1.2638 \mathrm{e}-01$ \\
\hline 0.7 & -0.0055 & -0.0942 & $8.8645 \mathrm{e}-02$ & 0.3227 & -0.0773 & $4.0001 \mathrm{e}-01$ \\
\hline 0.8 & 0.0670 & -0.0940 & $1.6093 \mathrm{e}-01$ & -0.0452 & -0.2229 & $1.7769 \mathrm{e}-01$ \\
\hline 0.9 & 0.3637 & -0.0937 & $4.5739 \mathrm{e}-01$ & 0.0634 & -0.2366 & $2.9998 \mathrm{e}-01$ \\
\hline
\end{tabular}

Table 4. Comparison of absolute errors of test problem 2 for different values of $k$ and $M$.

$\begin{array}{cr}B W S & \text { Maximum absolute er } \\ k=2, M=3 & 4.5739 \mathrm{e}-01 \\ k=3, M=2 & 4.0000 \mathrm{e}-01\end{array}$




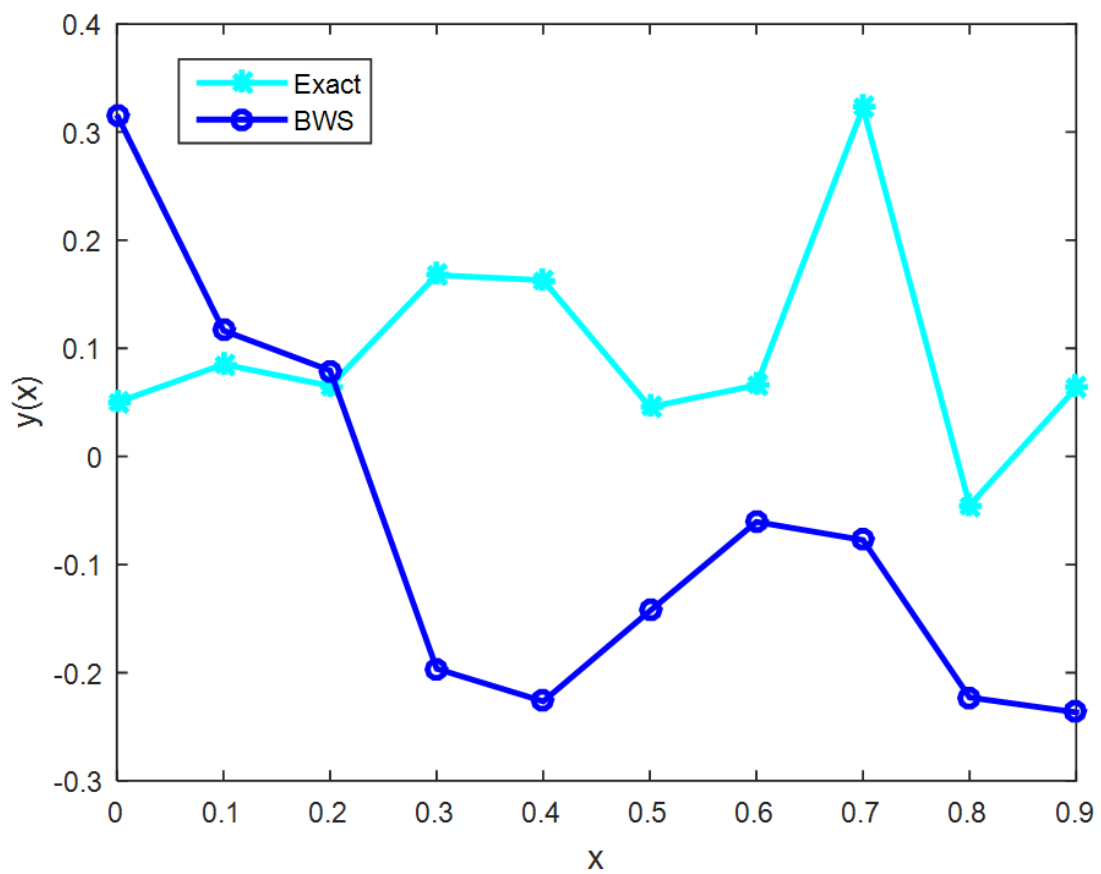

Figure 2. Graph of exact and BWS of test problem 2 for $k=3$ and $M=2$.

\section{Conclusion}

In this article, an effective strategy is provided to solve NSIVIE. This technique reduces these equations to a system of nonlinear algebraic equations with unknown Bernoulli coefficients, by using Bernoulli wavelets, their operational matrix of integration and stochastic operational matrix of integration which are solved by Newton's method. Error analysis of the proposed method is given. Moreover, the results obtained are compared with the exact solution with two Volterra integral equations and two NSIVIE in order to show the difference between the nonlinear Volterra integral equations and NSIVIE, and these examples show that the method described is precise and accurate and the results are in good agreement with the exact solution. By this we can conclude that the predicted algorithm is well organized and efficient.

\section{Acknowledgment}

First author is thankful to University Grants Commission (UGC), New Delhi, for supporting this work partially through UGC-SAP DRS-III for 2016-2021: F.510/3/DRSIII/2016 (SAP-I). 
Second author is thankful to Karnatak University Dharwad (KUD) for supporting this work under University Research Studentship (URS) 2016-2019: K. U. 40 (SC/ST) URS/2018-19/32/3/841 Dated: 07/07/2018.

\section{References}

[1] M. Khodabin, K. Maleknejad, M. Rostami and M. Nouri, Numerical approach for solving stochastic Volterra-Fredholm integral equations by stochastic operational matrix, Comput. Math. Appl. 64(6) (2012), 1903-1913.

https://doi.org/10.1016/j.camwa.2012.03.042

[2] M.H. Heydari, M.R. Hooshandasl, F.M. Maalek Ghaini and C. Cattani, A computational method for solving stochastic Itô-Volterra integral equations based on stochastic operational matrix for generalized hat basis functions, J. Comput. Phys. 270(1) (2014), 402-415. https://doi.org/10.1016/j.jcp.2014.03.064

[3] M.H. Heydari, C. Cattani, M.R. Hooshandasl and F.M. Maalek Ghaini, An efficient computational method for solving nonlinear stochastic Itô integral equations: Application for stochastic problems in physics, J. Comput. Phys. 283 (2015), 148-168. https://doi.org/10.1016/j.jcp.2014.11.042

[4] F. Mohammadi, A wavelet-based computational method for solving stochastic ItôVolterra integral equations, J. Comput. Phys. 298(1) (2015), 254-265.

https://doi.org/10.1016/j.jcp.2015.05.051

[5] K. Maleknejad, M. Khodabin and M. Rostami, Numerical solutions of stochastic Volterra integral equations by a stochastic operational matrix based on block pulse functions, Math. Comput. Modell. 55(3-4) (2012), 791-800.

https://doi.org/10.1016/j.mcm.2011.08.053

[6] K. Maleknejad, M. Khodabin and M. Rostami, A numerical method for solving mdimensional stochastic Itô-Volterra integral equations by stochastic operational matrix, Comput. Math. Appl. 63(1) (2012), 133-143.

[7] Y. Cao, D. Gillespie and L. Petzod, Adaptive explicit-implicit tau-leaping method with automatic tau selection, J. Chem. Phys. 126(22) (2007), 1-9.

https://doi.org/10.1063/1.2745299

[8] E. Platen and N. Bruti-Liberati, Numerical Solution of Stochastic Differential Equations with Jumps in Finance, Springer, Berlin, 2010.

https://doi.org/10.1007/978-3-642-13694-8

[9] M.H. Heydari, M.R. Hooshmandasl, A. Shakiba and C. Cattani, Legendre wavelets Galerkin method for solving nonlinear stochastic integral equations, Nonlinear Dyn. 
85(2) (2016), 1185-1202. https://doi.org/10.1007/s11071-016-2753-x

[10] M. Khodabin, K. Malekinejad, M. Rostami and M. Nouri, Numerical solution of stochastic differential equations by second order Runge-Kutta methods, Appl. Math. Modell. 53 (2011), 1910-1920. https://doi.org/10.1016/j.mcm.2011.01.018

[11] P.E. Kloeden and E. Platen, Numerical Solution of Stochastic Differential Equations, Springer, Berlin, 1999.

[12] J.C. Cortes, L. Jodar and L. Villafuerte, Numerical solution of random differential equations: a mean square approach, Math. Comput. Modell. 45(7-8) (2007), 757-765. https://doi.org/10.1016/j.mcm.2006.07.017

[13] B. Oksendal, Stochastic differential equations, $5^{\text {th }}$ ed., in: An Introduction with Applications, Springer, New York, 1998. https://doi.org/10.1007/978-3-662-03620-4_5

[14] H. Holden, B. Oksendal, J. Uboe and T. Zhang, Stochastic Partial Differential Equations, $2^{\text {nd }}$ ed., Springer, New York, 1998.

[15] A. Abdulle and A. Blumenthal, Stabilized multilevel Monte Carlo method for stiff stochastic differential equations, J. Comput. Phys. 251 (2013), 445-460. https://doi.org/10.1016/j.jcp.2013.05.039

[16] M.A. Berger and V.J. Mizel, Volterra equations with Itô integrals I, J. Integral Equations 2(3) (1980), 187-245.

[17] J.J. Levin and J.A. Nohel, On a system of integro-differential equations occurring in reactor dynamics, J. Math. Mech. 9 (1960), 347-368.

https://doi.org/10.1512/iumj.1960.9.59020

[18] M.H. Heydari, M.R. Hooshandasl, F.M. Maalek Ghaini and C. Cattani, A computational method for solving stochastic Itô-Volterra integral equations based on stochastic operational matrix for generalized hat basis functions, J. Comput. Phys. 270(1) (2014), 402-415. https://doi.org/10.1016/j.jcp.2014.03.064

[19] K. Maleknejad, M. Khodabin and M. Rostami, A numerical method for solving $m$ dimensional stochastic Itô-Volterra integral equations by stochastic operational matrix, Comput. Math. Appl. 63(1) (2012), 133-143.

[20] R.L. Stratonovich, A new representation for stochastic integrals and equations, SIAM J. Control. 4(2) (1966), 362-371. https://doi.org/10.1137/0304028

[21] F. Mirzaee and N. Samadyar, Application of operational matrices for solving system of linear Stratonovich Volterra integral equation, J. Comput. Appl. Math. 320 (2017), 164175. https://doi.org/10.1016/j.cam.2017.02.007 
[22] F. Mirzaee and N. Samadyar, On the numerical solution of stochastic quadratic integral equations via operational matrix method, Math. Methods Appl. Sci. 41(12) (2018), 44654479. https://doi.org/10.1002/mma.4907

[23] F. Mirzaee and N. Samadyar, Numerical solution of nonlinear stochastic Itô-Volterra integral equations driven by fractional Brownian motion, Math. Methods Appl. Sci. 41(4) (2018), 1410-1423. https://doi.org/10.1002/mma.4671

[24] Z. Taheri, S. Javadi and E. Babolian, Numerical solution of stochastic fractional integrodifferential equation by the spectral collocation method, J. Comput. Appl. Math. 321 (2017), 336-347. https://doi.org/10.1016/j.cam.2017.02.027

[25] F. Mirzaee and N. Samadyar, Application of Bernoulli wavelet method for estimating a solution of linear stochastic Itô-Volterra integral equations, Multidiscip. Model. Mater. Struct. 15(3) (2019), 575-598. https://doi.org/10.1108/MMMS-04-2018-0075

[26] E. Keshavarz, Y. Ordokhani and M. Razzaghi, Bernoulli wavelet operational matrix of fractional order integration and its applications in solving the fractional order differential equations, Appl. Math. Model. 38 (2014), 6038-6051.

https://doi.org/10.1016/j.apm.2014.04.064

[27] B.K. Mousavi, A. Askari Hemmat and M.H. Heydari, Wilson wavelets for solving nonlinear stochastic integral equations, Wavelets and Linear Algebra 4(2) (2017), 33-48.

This is an open access article distributed under the terms of the Creative Commons Attribution License (http://creativecommons.org/licenses/by/4.0/), which permits unrestricted, use, distribution and reproduction in any medium, or format for any purpose, even commercially provided the work is properly cited. 